which can be found in the cytoplasm of cells infected with this virus, have usually been refered to as "DNA factories". "They have now been rechristened in the Latin by Dahl and Kates (Virology, 42, 453; 1970), who apparently believe that virosomes is a better name because it does not "create a misleading impression concerning their composition and function". Be that as it may, Dahl and Kates have succeeded in isolating these complexes from infected HeLa cells. The virosomes are heterogeneous in size and density and the various classes of virosomes are related by some complex precursor product relationship which has yet to be fully resolved. What is more interesting is that the dense virosomes isolated three hours after infection contain nearly all the newly replicated viral DNA and an endogenous RNA polymerase activity (Dahl and Kates, ibid., 463). In vitro the isolated virosomes can synthesize RNA which competes in hybridization experiments with both "early" and "late" viral RNAs made in vivo.

To convince themselves of the reality of "early" and "late" viral RNAs in infected cells, Dahl and Kates transcribed in vitro purified viral DNA with RNA polymerase from $E$. coli. They then analysed the sequences present in RNA extracted from infected cells at early and late times by competition hybridization experiments with the RNA made in vitro by the bacterial enzyme. The RNA isolated from cells six hours after infection competed with about 97 per cent of the RNA made in vitro. By contrast the RNA extracted from cells $\mathbf{1 . 5}$ hours after infection only competed with about 40 per cent of the in vitro RNA. Apart from proving that sequences are present in "Iate" RNA which are not present in "early" RNA, this experiment indicates that $E$. coli RNA polymerase transcribes one and the same strand of vaccinia DNA as is transcribed in cells. Green and Westphal have respectively shown that $E$. coli polymerase asymmetrically transcribes the correct strand of adenovirus 2 and SV40 DNAs. Presumably the bacterial enzyme readily recognizes the promoter sequences in these viral DNAs, which hints that such sequences may be universally similar.

In the same issue of Virology (page 508), Bukrinskaya, Bykovsky and Zhdanov report that a ribonucleoprotein complex isolated from Sendai virus particles associates with ribosomes from Ehrlich ascites or chick embryo cells; and, in a suitable cell-free system, it stimulates a five-fold increase in the incorporation of leucine and phenylalanine. This stimulation is inhibited by puromycin, the omission of ATP or the S-100 fraction, and the extent of the incorporation depends on the amount of ribonucleoprotein complex added. Bukrinskaya et al. suggest, therefore, that they are detecting protein synthesis directed by Sendai RNA. They further claim that because the incubation mixture after thirty minutes contains an RNA polymerase activity, which is insensitive to actinomycin $\mathrm{D}$, one of the proteins being made is viral RNA polymerase. A full report of these experiments will be awaited with interest.

Jockusch, Bull and Kaesberg (ibid., 401) report that an $E$. coli cell-free system programmed with bacteriophage $Q \beta$ RNA makes three of the four proteins which are found in extracts of infected $E$. coli. This result, therefore, leaves open the question of whether the $Q \beta$ genome contains four rather than three cistrons, as does the characterization of $Q \beta$ mutants reported by Horiuchi and Matsuhashi (ibid., 49). They find that the twenty-two amber mutants which they have tested fit into only three complementation groups.

\section{BACTERIOPHAGE Heads and Tails}

\section{from our Cell Biology Correspondent}

THE assembly in vitro of such macromolecular complexes as bacteriophage R17, tobacco mosaic virus and ribosomes of $E$. coli from purified preparations of nucleic acids and proteins leaves little doubt about the importance of self assembly mechanisms in the genesis of viruses and cell organelles. But such observations can belie the amount of genetic information which may be required for the synthesis of comparatively simple macromolecular structures, for work with the DNA bacteriophages, such as the $\mathrm{T}$ phages and lambda phage, indicates that the number of genes implicated in the morphogenesis of these phage particles far exceeds the number of genes which specify molecules actually found in the particles.

Bacteriophage lambda, for example, is by any standards a simple structure; each particle consists of a head, the phage DNA and associated internal proteins enclosed in a roughly spherical protein coat, and a tail, an aggregate of protein. It is perhaps surprising therefore that genetic analyses of the lambda chromosome have implicated at least eighteen genes in the production of heads and tails, especially since Siminovitch's group (Virology, 42, 375, 390; 1970) have extended earlier work and proved that only three species of proteins account for at least 95 per cent of the total protein in each phage particle.

Buchwald, Steed-Glaister and Siminovitch, using two amber mutants, have isolated pure, empty heads which sediment at 114S and have a molecular weight of $18 \times 10^{6}$ and tails which sediment at $43 \mathrm{~S}$ and have a molecular weight of $6 \cdot 1 \times 10^{6}$. These two components account for about 75 per cent of the protein in the phages. Buchwald, Murialdo and Siminovitch have analysed on SDS polyacrylamide gels the proteins of dissociated heads, tails and whole phage. The head protein has a molecular weight of 37,500 , the tail protein has a molecular weight of 32,500 ; and a protein present in phage, but not in heads or tails, has a molecular weight of 11,000 . These three proteins account for 57, 19 and 19 per cent respectively of the total phage protein. Minor protein components no doubt account for the other 4-5 per cent-three to six minor proteins can be detected by electrophoresing dissociated whole phage.

Genetic analyses reported by others and now confirmed by Buchwald et al. show that the head protein is coded by gene $E$ while gene $V$ specifies the tail protein. The gene which specifies the third major protein, that with a molecular weight of 11,000 , has yet to be identified and the same is true of the genes which specify the minor protein components. If there are as many as six minor proteins specified by the phage, the roles of nine genes will be accounted for; but what do the other nine genes implicated in the morphogenesis of the phage do? Some at least may well specify proteins required for the assembly of the phage but not components of the particles. If that is the case it will be extremely difficult to define precisely the function of these putaive proteins, but their existence should caution against over-emphasis of the role of molecular self assembly. 